\title{
Anti-angiogenic Therapy for Advanced Primary Pulmonary Lymphoepithelioma-like Carcinoma: a Retrospective Multicenter Study
}

\section{Hejing Bao}

Southern Medical University Nanfang Hospital

\section{LingZhen $\mathrm{Ma}$}

Southern Medical University Nanfang Hospital

Mengge Yu

Southern Medical University Nanfang Hospital

Xiaoli Lin

Southern Medical University Nanfang Hospital

\section{Chengzhu Zhao}

Southern Medical University Nanfang Hospital

\section{Baishen Zhang}

Sun Yat-Sen University Cancer Prevention and Treatment Center: Sun Yat-sen University Cancer Center

\section{Juan Zhang}

nan fang yi ke da xue zhu jiang yi yuan: Zhujiang Hospital

\section{Guibao Peng}

Guangzhou Medical University

\section{Xiaotong Lin}

Guangzhou University of Chinese Medicine

\section{Yinhua Fang}

Chongqing University

\section{Hehong Bao}

Chongqing University

Shudong Ma ( $\sim$ mashudong@aliyun.com )

Southern Medical University Nanfang Hospital https://orcid.org/0000-0002-6090-9844

\section{Research Article}

Keywords: PPLELC, anti-angiogenic therapy, efficacy and safety, retrospective study

Posted Date: December 8th, 2021

DOI: https://doi.org/10.21203/rs.3.rs-1109138/v1

License: (c) (i) This work is licensed under a Creative Commons Attribution 4.0 International License. Read Full License 


\section{Abstract}

Purpose: Primary pulmonary lymphoepithelioma-like carcinoma (PPLELC) is a rare subtype of primary non-small cell lung cancer (NSCLC). Currently, there is still lack of research data on anti-angiogenic therapy of advanced PPLELC. The purpose of this study was to investigate the efficacy and safety of anti-angiogenic therapy combined with chemotherapy compared with traditional chemotherapy for these patients.

Methods: Advanced PPLELC patients admitted to six grade A hospitals from January 2013 to January 2021 were selected. The patients received anti-angiogenic therapy combined with chemotherapy(AT group) or chemotherapy (CT group)alone.

Results: A total of 65 patients were enrolled in this study, including 31 patients in the AT group treated with antiangiogenic therapy combined with chemotherapy, and 34 patients in the CT group treated with chemotherapy alone. As of October 1, 2021, the median progression-free survival in the AT group was 11.2 months [95\% confidence interval (Cl), 5.9-16.5], The median progression-free survival in the CT group was 7.0 months [95\%Cl, 5.1-8.9][Hazard Ratio (HR), 0.49; $95 \% \mathrm{Cl}, 0.29-0.83 ; \mathrm{P}=0.008]$. The 1 -year PFS rates were $41.9 \%$ and $17.6 \%$, respectively. The ORR of two groups were $45.2 \%$ (95\% $\mathrm{Cl}, 0.27$ to 0.64$), 38.2 \%(95 \% \mathrm{Cl}, 0.21$ to 0.56$),(\mathrm{P}=0.571)$. The $\mathrm{DCR}$ of two groups were $93.5 \%(95 \% \mathrm{Cl}, 0.84$ to 1.03), $88.2 \%(95 \% \mathrm{Cl}, 0.77$ to 1.00$),(P=0.756)$.

Conclusions: Among patients with advanced PPLELC, the progression free survival of patients with anti-angiogenic therapy combined with chemotherapy is better than that of patients with chemotherapy alone. Anti-angiogenic therapy combined with chemotherapy is an optional treatment scheme.

\section{Introduction}

Lymphoepitheliomatoid carcinoma is a rare epithelial neoplasm originating mostly in the nasopharynx and also in the foregut $^{12}$. Primary pulmonary lymphoepithelioma-like carcinoma is a rare subtype of primary non-small cell lung cancer that histologically resembles undifferentiated nasopharyngeal carcinoma (NPC) ${ }^{3}$, the incidence in all cases of non-small cell lung cancer is about $0.7 \%^{24}$. It was previously classified as a variant of large cell carcinoma ${ }^{5}$, then reclassified as other and unclassified cancers in the world Health Organization (WHO) Classification of Lung tumors in $2015^{6}$.

First described by Begin et al. In 1987, PPLELC has been considered to be closely associated with epstein-Barr virus (EBV) infection ${ }^{8}$. About 1,600 cases have been reported worldwide in the past 33 years since the discovery ${ }^{49-13}$, mainly focused on the past five years, reporting mainly in Asia, especially Hong Kong, Taiwan, Guangdong and other regions. PPLELC usually affects never smokers, is gender-neutral, and is younger than non-small cell lung cancer ${ }^{14}$. Similar to other types of lung cancer, the prognosis is good for most patients diagnosed early with lung lymphoepithelioid, with a median overall survival of approximately 107 months and a 5-year survival of approximately $60 \%$, compared with a mean survival of 13 months for patients with non-lung lymphoepithelioid ${ }^{1516}$.

The main treatment strategy for early PPLELC is surgery ${ }^{1417}$. For patients with distant metastasis, surgical treatment is not possible, and a variety of treatment modes are usually accepted, including chemotherapy, radiotherapy, and immunotherapy ${ }^{491018-22}$. Several treatments have been reported in the literature, but the treatment of PPLELC is empirical due to its rarity. However, there is still a lack of research data on anti-angiogenic therapy in advanced PPLELC. Therefore, this study aims to investigate the effect of anti-angiogenic therapy on advanced mPPLELC patients.

\section{Materials And Methods}




\section{Patients}

Patients aged 18 years or older, stage IV, diagnosed as lung lymphoepithelioid carcinoma according to the 2015 WHO histological classification criteria for lung tumors, and without allergenic EGFR or ALK mutations; According to the Solid Tumor Response Assessment Criteria (RECIST), version 1.1 has at least one measurable lesion. This retrospective study collected data of PPLELC patients from Nanfang Hospital affiliated to Southern Medical University, Zhujiang Hospital affiliated to Southern Medical University, The First Affiliated Hospital of Guangzhou University of Traditional Chinese Medicine, Cancer Hospital of Sun Yat-sen University, Cancer Hospital affiliated to Guangzhou Medical University, Three Gorges Hospital affiliated to Chongqing University in Guangdong Province from January 2013 to January 2021. 65 patients with advanced PPLELC were eventually included in the study. (figure1)

\section{Experimental design and treatment}

In this real-world retrospective study, patients were divided into AT group treated with anti-angiogenic therapy combined with chemotherapy and CT group treated with chemotherapy. AT group received anti-angiogenic therapy, including bevacizumab( $n=22)$, endu( $(n=6)$ or arotinib $(n=3)$, the chemotherapy regimen included paclitaxel $(n=14)$, pemetrexed $(n=10)$ or gemcitabine $(n=7)$ combined with platinum or mono-therapy. The chemotherapy regimen in CT group included paclitaxel( $(=19)$, pemetrexed( $(n=10)$ or gemcitabine $(n=5)$ combined with platinum or mono-therapy.

\section{Assessment}

The tumor response assessment program is conducted every two to four cycles. Tumor response was assessed as complete response (CR), partial response (PR), disease stability (SD), or disease progression (PD) according to RECIST version 1.1. Adverse events and laboratory abnormalities were graded according to the National Cancer Institute Standard for Common Terminology for Adverse Events, Version 4.0.

\section{Statistical analysis}

Efficacy was assessed in the enrolled population and safety was assessed in the treated population. Kaplan-meier methods were used to estimate overall survival and progression-free survival. T test was used for measurement data (age) and Chi-square test (Cartesian test) was used for counting data. All statistical analyses were performed in IBM SPSS Statistics(Version 19.0, Armonk, NY, USA). When P value was less than 0.05 , the difference was considered statistically significant.

\section{Results}

\section{Patient characteristics}

The median age of patients was 54 years (48-62 years). There were 38 female patients (58.5\%) and 27 male patients (41.5\%). ECOG score was 0 in 24 patients (36.9\%) and 1 in 41 patients (63.1\%). 18 patients (27.7\%) were smokers, and 10 patients $(15.4 \%)$ had a family history. There were 10 patients $(15.4 \%)$ in stage III and 55 patients $(84.6 \%)$ in stage IV. Patients with single organ metastasis accounted for 19 (29.2\%), and patients with multiple organ metastasis accounted for 46 (70.8\%). The most common tumor sites were middle lobe of right lung and lower lobe of right lung, with 18 $(27.7 \%)$ and $17(26.1 \%)$ patients, respectively. There were 61 patients (95.3\%) who were positive for Epstein-Barr virus, 15 patients $(23.1 \%)$ had received radiotherapy, and 4 patients $(6.2 \%)$ with gene mutations, including 3 gene mutations in the AT group, which were NRAS Q61L(14.5\%) missense mutation, SMAD4 K110Ter(19.5\%) mutation and FGFR3TACC3(F17:T10) fusion. MYC amplification was found in 1 case in CT group. There was no significant difference between the two groups at baseline $(P>0.05)$. (Table1) 
Table 1

Baseline characteristics

\begin{tabular}{|c|c|c|c|c|}
\hline & $\begin{array}{l}\text { All patients } \\
(n=65)\end{array}$ & $\begin{array}{l}\text { AT group } \\
(n=31)\end{array}$ & $\begin{array}{l}\text { CT group } \\
(n=34)\end{array}$ & $P$ \\
\hline Age, years Median(IQR) & $54(48-62)$ & $55(48.5-61.5)$ & $54(48-61.25)$ & 0.892 \\
\hline Sex & & & & 0.285 \\
\hline Female & $27(41.5 \%)$ & $15(48.4 \%)$ & $12(35.3 \%)$ & \\
\hline Male & $38(58.5 \%)$ & $16(51.6 \%)$ & $22(64.7 \%)$ & \\
\hline ECOG performance status & & & & 0.592 \\
\hline 0 & $24(36.9 \%)$ & $12(38.7 \%)$ & $11(32.3 \%)$ & \\
\hline 1 & $41(63.1 \%)$ & $19(61.3 \%)$ & $23(67.6 \%)$ & \\
\hline Smokers & $18(27.7 \%)$ & $10(32.3 \%)$ & $8(23.5 \%)$ & 0.432 \\
\hline Family history & $10(15.4 \%)$ & $3(9.7 \%)$ & $7(20.6 \%)$ & 0.309 \\
\hline TNM & & & & 0.382 \\
\hline III & $10(15.4 \%)$ & $3(9.7 \%)$ & $7(20.6 \%)$ & \\
\hline IV & $55(84.6 \%)$ & $28(90.3 \%)$ & $27(79.4 \%)$ & \\
\hline Metastases & & & & 0.260 \\
\hline single organ & $19(29.2 \%)$ & $7(22.6 \%)$ & $12(35.3 \%)$ & \\
\hline multiple organ & $46(70.8 \%)$ & $24(77.4 \%)$ & $22(64.7 \%)$ & \\
\hline Tumor location & & & & 0.903 \\
\hline left upper lobe of lung & $10(15.4 \%)$ & $5(16.1 \%)$ & $5(14.7 \%)$ & \\
\hline left lower lobe of lung & $15(23.1 \%)$ & $8(25.8 \%)$ & $7(20.6 \%)$ & \\
\hline right upper lobe of lung & $5(7.7 \%)$ & $2(6.5 \%)$ & $3(8.8 \%)$ & \\
\hline right middle lobe of lung & $18(27.7 \%)$ & $7(22.6 \%)$ & $11(32.4 \%)$ & \\
\hline right lower lobe of lung & $17(26.1 \%)$ & $9(29.0 \%)$ & $8(23.5 \%)$ & \\
\hline $\mathrm{EB}(+)$ & $61(95.3 \%)$ & $29(93.5 \%)$ & $32(94.1 \%)$ & 1.0 \\
\hline Radiotherapy & $15(23.1 \%)$ & $6(19.3 \%)$ & $9(26.5 \%)$ & 0.496 \\
\hline Gene mutation & $4(6.2 \%)$ & $3(9.7 \%)$ & $1(2.9 \%)$ & 0.540 \\
\hline
\end{tabular}

\section{Efficacy}

The minimum follow-up time was about 1.4 months. Median follow-up time was 18 months in the AT group (95\%Cl, 13.526) and 19 months in the CT group $(95 \% \mathrm{Cl}, 13-28)$. AT the end of the follow-up period, 11 patients (35.5\%) in the AT group and 12 patients (35.3\%) in the CT group continued treatment. Anti-angiogenic therapy was administered either as 
up-front or salvage therapy (1st-line $n=18, \geq 2 n d$-line $n=13$, cross-line $n=7$ ), while chemotherapy was used in the 1 st-line therapy.

There were no CR cases in both AT group and CT group. In AT group, 14 patients (45.2\%) reached PR, 15 patients (48.3\%) reached SD, and 2 patients (6.5\%) did not respond to treatment. In CT group, 13 patients (38.2\%) reached PR, 17 patients (50.0\%) reached SD, and 4 patients (11.8\%) did not respond to treatment. The ORR of AT group and CT group were 14 $(45.2 \%, 0.27-0.64)$ and $13(38.2 \%, 0.21-0.56), P=0.571 ; D C R$ were $29(93.5 \%, 0.84-1.03)$ and $30(88.2 \%, 0.77-1.00), P=$ 0.756. (Table2)(figure2)

Table 2

Efficacy of treatments

\begin{tabular}{|llll|}
\hline & AT group(n=31) & CT group(n=34) & P \\
\hline Best overall response & & & 0.763 \\
\hline Complete response & 0 & $13(38.2 \%)$ \\
\hline Partial response & $14(45.2 \%)$ & $17(50.0 \%)$ & 0.571 \\
\hline Stable disease & $15(48.3 \%)$ & $4(11.8 \%)$ & 0.756 \\
\hline Progressive disease & $2(6.5 \%)$ & $13(38.2 \%, 0.21-0.56)$ & $30(88.2 \%, 0.77-1.00)$ \\
\hline Objective response & $14(45.2 \%, 0.27-0.64)$ & $29(93.5 \%, 0.84-1.03)$ & \\
\hline Disease control & $2 \%$ the & \\
\hline $\begin{array}{l}\text { Data are } n \text { (\%). Confirmed complete and partial responses were assessed by the investigator according to the } \\
\text { Response Evaluation Criteria in Solid Tumors, version 1.1. }\end{array}$
\end{tabular}

The median progression free survival was 11.2 months in the AT group [95\% Cl, 5.9-16.5] and 7.0 months in the CT Group [95\% Cl, 5.1-8.9] [HR, 0.49; 95\% Cl, 0.29-0.83; $\mathrm{P}=0.008]$. The 1-year PFS rates were $41.9 \%$ and $17.6 \%$ respectively.

(figure3)

In this study, we reported a 59-year-old female patient with FGFR3-TACC3 (F17:T10) fusion, no smoking history, tumor located in the right lung with lymphatic, liver, and bone metastases, clinical stage T4N3M1, pathologically confirmed as PPLELC, Genetic analysis revealed FGFR3-TACC3 (F17:T10) fusion without EGFR, ALK and other conventional mutations. The patient was treated with androtinib in combination with albumin-paclitaxel and carboplatin at the physician's recommendation, achieving disease control and PFS for 8 months. (figure4)

\section{Safety}

Treatment-related adverse events (both hematological and non-hematological poisoning events) occurred more frequently in the AT group than in the CT group. In the AT group, there were 17 cases $(54.8 \%)$ of grade 3 and 8 cases $(25.8 \%)$ of grade 4 treatment-related adverse events. There were 13 cases (38.2\%) of grade 3 and 12 cases (35.3\%) of grade 4 treatment-related adverse reactions in the CT group. The most common adverse reactions in AT group were decreased appetite 7 (22.6\%), anemia 8 (25.8\%), leukopenia 14 (45.2\%), neutropenia 16 (51.6\%), and decreased platelet 8 (25.8\%). Anemia 10 (29.4\%), leukopenia 14 (41.2\%), neutropenia 15 (44.1\%), and thrombocytopenia 10 (29.4\%) were most common in the CT group. (Table3) 
Table 3

Treatment-related adverse events

\begin{tabular}{|c|c|c|c|c|c|c|c|c|}
\hline & \multicolumn{4}{|c|}{ AT group $(n=31)$} & \multicolumn{4}{|c|}{ CT group $(n=34)$} \\
\hline & Grade1-2 & Grade3 & Grade4 & ALL & Grade1-2 & Grade3 & Grade4 & ALL \\
\hline Fatigue & $4(12.9 \%)$ & 0 & 0 & $4(12.9 \%)$ & $2(5.9 \%)$ & 0 & 0 & $2(5.9 \%)$ \\
\hline Nausea & $6(19.3 \%)$ & 0 & 0 & 6(19.3\%) & $5(14.7 \%)$ & 0 & 0 & $5(14.7 \%)$ \\
\hline Vomiting & $4(12.9 \%)$ & 0 & 0 & $4(12.9 \%)$ & $4(11.8 \%)$ & 0 & 0 & $4(11.8 \%)$ \\
\hline Anorexia & $7(22.6 \%)$ & 0 & 0 & $7(22.6 \%)$ & $6(17.6 \%)$ & 0 & 0 & $6(17.6 \%)$ \\
\hline $\begin{array}{l}\text { Peripheral } \\
\text { sensory } \\
\text { neuropathy }\end{array}$ & $2(6.5 \%)$ & 0 & 0 & $2(6.5 \%)$ & $2(5.9 \%)$ & 0 & 0 & $2(5.9 \%)$ \\
\hline Hypertension & $2(6.5 \%)$ & $2(6.5 \%)$ & 0 & $4(12.9 \%)$ & 0 & 0 & 0 & 0 \\
\hline Proteinuria & $2(6.5 \%)$ & 0 & 0 & $2(6.5 \%)$ & 0 & 0 & 0 & 0 \\
\hline Anemia & $8(25.8 \%)$ & 0 & 0 & $8(25.8 \%)$ & $10(29.4 \%)$ & 0 & 0 & $10(29.4 \%)$ \\
\hline $\begin{array}{l}\text { White blood } \\
\text { cell } \\
\text { decreased }\end{array}$ & 10(32.3\%) & $4(12.9 \%)$ & 0 & $14(45.2 \%)$ & $9(26.4 \%)$ & $2(5.9 \%)$ & $3(8.8 \%)$ & $14(41.2 \%)$ \\
\hline $\begin{array}{l}\text { Neutrophil } \\
\text { count } \\
\text { decreased }\end{array}$ & $2(6.5 \%)$ & $6(19.3 \%)$ & $8(25.8 \%)$ & $16(51.6 \%)$ & $3(8.8 \%)$ & $3(8.8 \%)$ & $9(26.4 \%)$ & $15(44.1 \%)$ \\
\hline $\begin{array}{l}\text { Lymphocyte } \\
\text { count } \\
\text { decreased }\end{array}$ & $4(12.9 \%)$ & $2(6.5 \%)$ & & $6(19.3 \%)$ & $3(8.8 \%)$ & $3(8.8 \%)$ & 0 & $6(17.6 \%)$ \\
\hline $\begin{array}{l}\text { platelet } \\
\text { count } \\
\text { decreased }\end{array}$ & $6(19.3 \%)$ & $2(6.5 \%)$ & 0 & $8(25.8 \%)$ & $6(17.6 \%)$ & $4(11.8 \%)$ & 0 & $10(29.4 \%)$ \\
\hline $\begin{array}{l}\text { ALT } \\
\text { increased }\end{array}$ & $2(6.5 \%)$ & 0 & 0 & $2(6.5 \%)$ & 0 & $1(2.9 \%)$ & 0 & $1(2.9 \%)$ \\
\hline $\begin{array}{l}\text { AST } \\
\text { increased }\end{array}$ & $2(6.5 \%)$ & 0 & 0 & $2(6.5 \%)$ & $2(5.9 \%)$ & 0 & 0 & $2(5.9 \%)$ \\
\hline $\begin{array}{l}\text { Blood } \\
\text { bilirubin } \\
\text { increased }\end{array}$ & $2(6.5 \%)$ & 0 & 0 & $2(6.5 \%)$ & $2(5.9 \%)$ & 0 & 0 & $2(5.9 \%)$ \\
\hline $\begin{array}{l}\text { Rash } \\
\text { maculo- } \\
\text { papular }\end{array}$ & $5(16.1 \%)$ & 0 & 0 & $5(16.1 \%)$ & $2(5.9 \%)$ & 0 & 0 & $2(5.9 \%)$ \\
\hline $\begin{array}{l}\text { Tracheal } \\
\text { fistula }\end{array}$ & 0 & $1(3.2 \%)$ & 0 & $1(3.2 \%)$ & 0 & 0 & 0 & 0 \\
\hline Overall & 68 & 17 & 8 & 93 & 56 & 13 & 12 & 81 \\
\hline
\end{tabular}

\section{Discussion}

PPLELC is a rare subtype of NSCLC. In this era of targeted therapy and checkpoint inhibitors for non-small cell lung cancer, anti-angiogenic drugs still play an important role ${ }^{23}$. However, the prognosis of advanced PPLELC is poor, due to 
the lack of drug treatment data, the role of anti-angiogenic therapy in PPLELC is unknown. It is worth exploring the effect of anti-angiogenic therapy in PPLELC.

Induction of angiogenesis is one of the 10 characteristics of malignant tumors. Angiogenesis provides essential nutrients for tumor growth and is an important prerequisite for tumor hematogenous dissemination. The ECOG-4599 study established the status of bevacizumab combined with first-line chemotherapy ${ }^{24}$. Small molecule TKI drugs targeting angiogenesis have become one of the research hotpots in recent years. The biggest feature of small molecule drugs is that their target coverage is more comprehensive. In addition to VEGF/R and other pathways, they can also cover PDGF/R, FGF/FGFR and other pathways.

In this study, a patient with FGFR3-TACC3 fusion was reported. In general, operable FGFR3 gene fusion is found relatively commonly in glioma and bladder cancer ${ }^{25}$, FGFR3-TACC3 fusion has been reported in $2.5 \%$ of NPC ${ }^{26}$. However, FGFR3 alteration was rarely observed in NSCLC, and was detected in $0.1 \%$ of adenocarcinomas and $0.6 \%$ of squamous cell carcinomas, respectively ${ }^{2728}$. The prevalence of FGFR3 in LELC was $4 \%{ }^{29}$. FGFR3-TACC3 is reported to be a relapsing drug resistance mechanism that can bypass EGFR blockade by all generations of EGFR TKI in NSCLC ${ }^{30}$. In this study, FGFR3-TACC3 fusion patients were treated with small molecule multi-target TKI, which inhibited VEGFR, PDGFR, FGFR and c-Kit targets, showing anti-tumor angiogenesis and tumor growth inhibition. PFS benefits were obtained in combination with chemotherapy, suggesting that FGFR3 aberrations may represent an opportunity to target therapy in PPLELC .

However, anti-angiogenic therapy alone does not significantly improve patient outcomes, as the removal of blood vessels transforms tumor cells into a hypoxic-tolerant phenotype. Combination of anti-angiogenic therapy with other therapies, including chemotherapy, radiotherapy, immunotherapy and anti-epidermal growth factor receptor (EGFR) therapy, has good efficacy due to the vasonormalization effect induced by anti-angiogenic agents ${ }^{31}$. Radiation alone as the sole means of treating cancer often triggers the angiogenesis pathway, leading to upregulation of multiple angiogenic growth factors, and anti-angiogenic inhibitors can help overcome this fact ${ }^{32}$. Randomized Phase III studies have also shown that treatment with antiangiogenic agents in combination with PD-L1 antibodies significantly improves survival compared with standard therapy in RCC, NSCLC and HCC. However, there are still many problems to be solved, such as how to choose a more reasonable combination partner for anti-angiogenic therapy, the timing, recommended dosage and the proportion of anti-angiogenic inhibitor combination therapy ${ }^{31}$. In this study, anti-angiogenic therapy combined with chemotherapy in patients with advanced PPLELC was included. The results showed that compared with chemotherapy alone, it can improve the short-term efficacy of patients. It is well known that immunotherapy can improve the long-term prognosis of patients. If anti-angiogenic combined with immunotherapy and chemotherapy is a better choice, it is worth further exploration.

Our study found that anti-angiogenic therapy combined with chemotherapy was more beneficial than chemotherapy alone in patients with advanced PPLELC, but there was no way to screen the dominant population, and some patients did not respond to anti-angiogenic therapy combined with chemotherapy. Both targeted therapy and immunotherapy have corresponding markers to accurately identify the population, but there is no recognized marker in the field of antiangiogenic therapy. Bevacizumab is a monoclonal antibody with a clear target of VEGF, so it is logical that VEGF expression might predict benefit. However, clinical studies have found that VEGF expression does not predict the benefit of bevacizumab addition ${ }^{33}$. The tumor vasculature is a target for antiangiogenic drugs, but quantitative or qualitative measures of the vasculature cannot be used as biomarkers of efficacy because there is no standardized method for measuring microvessel density and there is considerable potential for selection and observer bias. The detection of vascular phenotype and tumor cell genotype is still in the stage of preclinical research ${ }^{34}$. Compared to using the tissue biomarkers to select effective anti-angiogenic drugs, the studies of discover and validation in the non-invasive, dynamic 
biomarker were at a more advanced stage, including measuring the circulating protein related to angiogenesis, circulating micrornas, secrete body outside, circulating endothelial cells and or estimates of progenitor cells and vascular imaging ${ }^{35}$.

Our study has some limitations. This study was from a retrospective cohort and the number of included patients was limited. This is due to the rarity of PPLELC and the small number of patients, even fewer patients in the advanced stage of metastasis. Moreover, the emergence and application of different anti-angiogenic drugs in clinical practice will affect the results. Differences in patients' chemotherapy regimens also affected the results. However, this study firstly reported the value of anti-angiogenic therapy in advanced PPLELEC. Data were collected from multiple centers in this study, and patients came from areas with high PPLELC incidence, which has certain advantages.

In conclusion, compared with the current treatment of patients with advanced PPLELC, anti-angiogenic therapy is clinically beneficial and sufficiently safe, and the number of patients should be further expanded or the follow-up period extended to further observe the survival benefit.

\section{Declarations}

\section{Data availability}

Datasets generated and analyzed during the study are available from HB on reasonable request.

\section{Code availability}

Not applicable.

\section{Acknowledgements}

The authors are grateful to all staff at the study centre who contributed to this study.

\section{Funding}

This work was supported by the Antitumor Angiogenesis Targeted Therapy Research Fund of the Chinese Society of Clinical Oncology (Y-S2016-004), the Nanfang Hospital Dean's Fund (2018B013), and the Wu Jieping Medical Foundation (320.6750.19061)

\section{Contributions}

HJB: Conceptualization; Data curation; Resources; Writing-original draft; Writing-review \& editing. LZM,MGY,LXL,CZZ: Methodology; Visualization; Writing-review \& editing. BSZ,JZ,GBP,XTL: Resources; Validation; Visualization. YHF,HHB: Formal analysis; Methodology; Software; Writing-original draft. SDM: Funding acquisition; Project administration; Supervision; Visualization. All authors read and approved the final manuscript.

\section{Ethics declarations}

\section{Conflict of interest}

All authors declare no conflicts of interest associated with this manuscript.

\section{Ethics approval}


Because of the nature of retrospective design and patient anonymization, the ethical board of the Institutional Ethics Committee of the Southern medical university Nanfang Hospital approved the retrospective study.

\section{Consent to participate}

The need for written informed consent was waived due to the retrospective nature of the study.

\section{Consent for publication}

All authors have consented to publication of the results presented in this manuscript.

\section{References}

1. Ambrosio MR, Rocca BJ, Onorati M, et al. Lymphoepithelioma-Like Carcinoma of the Ovary. Int J Surg Pathol. 2011;19(4):514-517.

2. Anand A, Zayac A, Curtiss C, Graziano S. Pulmonary Lymphoepithelioma-like Carcinoma Disguised as Squamous Cell Carcinoma. J Thorac Oncol. 2018;13(5):E75-E76.

3. Hayashi T, Haba R, Tanizawa J, et al. Cytopathologic Features and Differential Diagnostic Considerations of Primary Lymphoepithelioma-Like Carcinoma of the Lung. Diagn Cytopathol. 2012;40(9):820-825.

4. Xie Z, Liu L, Lin X, et al. A multicenter analysis of genomic profiles and PD-L1 expression of primary lymphoepithelioma-like carcinoma of the lung. Modern Pathol. 2020;33(4):626-638.

5. Travis WD, Brambilla E, Müller-Hermelink HK, Harris CC. Pathology and genetics of tumours of the lung, pleura, thymus and heart. 2004.

6. Available N. Pathology and Genetics of Tumours of the Lung, Pleura, Thymus and Heart (IARC/World Health Organization Classification of Tumours). Intl Agency for Research on Cancer. 2015.

7. Begin LR, Eskandari J, Joncas J, Panasci L. Epstein-Barr virus related lymphoepithelioma-like carcinoma of lung. J Surg Oncol. 1987;36(4):280-283.

8. Han AJ, Xiong M, Zong YS. Association of Epstein-Barr virus with lymphoepithelioma-like carcinoma of the lung in southern China. Am J Clin Pathol. 2000;114(2):220-226.

9. Kim C, Rajan A, Debrito PA, Giaccone G. Metastatic lymphoepithelioma-like carcinoma of the lung treated with nivolumab: a case report and focused review of literature. Translational lung cancer research. 2016-01-01 2016;5(6):720-726.

10. Darrason M, Martin A, Soussan M, et al. Immunotherapy for LELC: Case Report and a Focused Review. Clin Lung Cancer. 2019;20(3):e393-e401.

11. Hong S, Liu D, Luo S, et al. The genomic landscape of Epstein-Barr virus-associated pulmonary lymphoepitheliomalike carcinoma. Nat Commun. 2019;10(3108).

12. Wu Q, Wang W, Zhou P, et al. Primary pulmonary lymphoepithelioma-like carcinoma is characterized by high PD-L1 expression, but low tumor mutation burden. Pathology - Research and Practice. 2020;216(8):153043.

13. Chen J, Gu C, Chen X, et al. Clinicopathological and prognostic analyses of 86 resected pulmonary lymphoepithelioma-like carcinomas. J Surg Oncol. 2020.

14. Lin Z, Situ D, Chang X, et al. Surgical treatment for primary pulmonary lymphoepithelioma-like carcinoma. Interact Cardiov Th. 2016;23(1):41-46.

15. He J, Shen J, Pan H, Huang J, Liang W, He J. Pulmonary lymphoepithelioma-like carcinoma: a Surveillance, Epidemiology, and End Results database analysis. J Thorac Dis. 2015;7(12):2330-2338. 
16. Liang Y, Wang L, Zhu Y, et al. Primary pulmonary lymphoepithelioma-like carcinoma. Cancer-Am Cancer Soc. 2012;118(19):4748-4758.

17. Ho JC, Wong MP, Lam WK. Lymphoepithelioma-like carcinoma of the lung. Respirology. 2006;11(5):539-545.

18. Kumar V, Dave V, Harris J, Huang Y. Response of advanced stage recurrent lymphoepithelioma-like carcinoma to nivolumab. Immunotherapy-Uk. 2017;9(12):955-961.

19. Narayanan A, Knollmann FD, Walby JAS, Lim S, Gandara DR, Riess JW. EBV-positive Primary Pulmonary Lymphoepithelioma-like Carcinoma Response to PD-L1 Blockade. Clin Lung Cancer. 2019;20(3):e238-e241.

20. Zhou N, Lin Y, Peng X, Wang Y, Wang Y. Thorough survey and analysis of pulmonary lymphoepithelioma-like carcinoma in Macau and multimodality treatment for advanced disease. Lung Cancer. 2019;138:116-123.

21. Tang Z, Fang R, Tong G, Liu P, Ou ZA, Tang Y. Overcoming resistance to anti-PD-1 immunotherapy in lymphoepithelioma-like carcinoma: A case report and review of the literature. Lung Cancer. 2020;146:335-340.

22. Qiu Z, Zhou P, Wang K. Primary Pulmonary Lymphoepithelioma-Like Carcinoma Response Favorably To Nivolumab: A Case Report. Oncotargets Ther. 2019;12:8595-8600.

23. Shukla NA, Yan MN, Hanna N. The Story of Angiogenesis Inhibitors in Non-small-cell Lung Cancer: The Past, Present, and Future. Clin Lung Cancer. 2020-07-01 2020;21(4):308-313.

24. Sandler A, Gray R, Perry MC, et al. Paclitaxel-carboplatin alone or with bevacizumab for non-small-cell lung cancer. $N$ Engl J Med. 2006-12-14 2006;355(24):2542-2550.

25. Wu YM, Su F, Kalyana-Sundaram S, et al. Identification of targetable FGFR gene fusions in diverse cancers. Cancer Discov. 2013-06-01 2013;3(6):636-647.

26. Yuan L, Liu ZH, Lin ZR, Xu LH, Zhong Q, Zeng MS. Recurrent FGFR3-TACC3 fusion gene in nasopharyngeal carcinoma. Cancer Biol Ther. 2014-01-20 2014;15(12):1613-1621.

27. F A, M A, AS B. AACR Project GENIE: Powering Precision Medicine through an International Consortium. Cancer Discov. 2017-08-01 2017;7(8):818-831.

28. Qin A, Johnson A, Ross JS, et al. Detection of Known and Novel FGFR Fusions in Non-Small Cell Lung Cancer by Comprehensive Genomic Profiling. J Thorac Oncol. 2019-01-01 2019;14(1):54-62.

29. Chau SL, Tong JH, Chow C, et al. Distinct Molecular Landscape of Epstein-Barr Virus Associated Pulmonary Lymphoepithelioma-Like Carcinoma Revealed by Genomic Sequencing. Cancers (Basel). 2020-07-27 2020;12(8).

30. Ou SI, Horn L, Cruz M, et al. Emergence of FGFR3-TACC3 fusions as a potential by-pass resistance mechanism to EGFR tyrosine kinase inhibitors in EGFR mutated NSCLC patients. Lung Cancer. 2017-09-01 2017;111:61-64.

31. Tian W, Cao C, Shu L, Wu F. Anti-Angiogenic Therapy in the Treatment of Non-Small Cell Lung Cancer. Onco Targets Ther. 2020-01-20 2020;13:12113-12129.

32. Rani V, Prabhu A. Combining Angiogenesis Inhibitors with Radiation: Advances and Challenges in Cancer Treatment. Curr Pharm Des. 2021-01-20 2021;27(7):919-931.

33. Otrock ZK, Hatoum HA, Musallam KM, Awada AH, Shamseddine Al. Is VEGF a predictive biomarker to antiangiogenic therapy? Crit Rev Oncol Hematol. 2011-08-01 2011;79(2):103-111.

34. Jubb AM, Oates AJ, Holden S, Koeppen H. Predicting benefit from anti-angiogenic agents in malignancy. Nat Rev Cancer. 2006-08-01 2006;6(8):626-635.

35. Di Paolo V, Colletti M, Ferruzzi V, et al. Circulating Biomarkers for Tumor Angiogenesis: Where Are We? Curr Med Chem. 2020-01-20 2020;27(14):2361-2380.

\section{Figures}




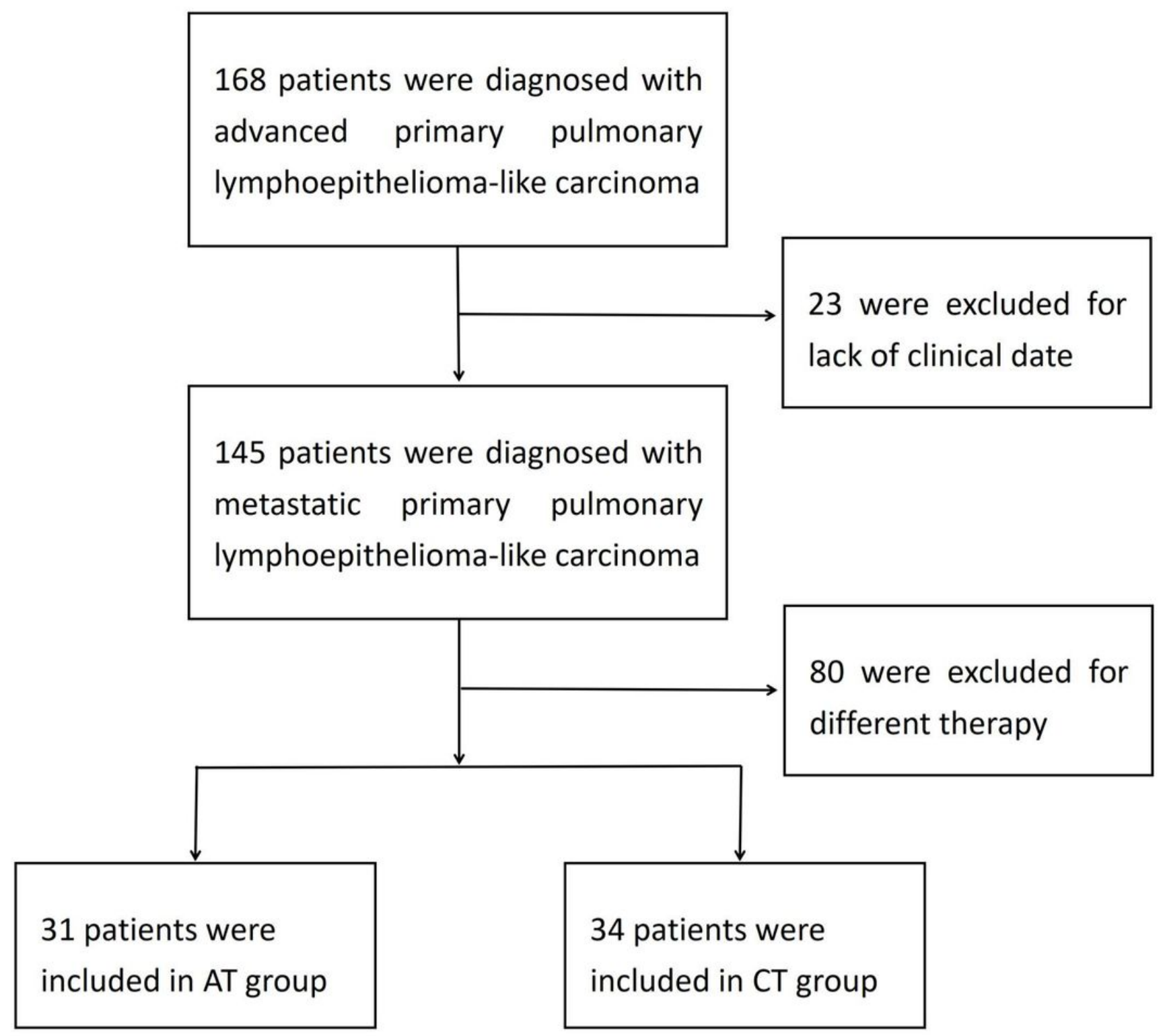

Figure 1

Study enrolment. A total of 168 patients diagnosed with advanced PPLELC in six grade A hospitals from January 2013 to January 2021 were selected, and 65 patients were eventually included. Due to the lack of clinical data, 23 patients were excluded. 80 patients were excluded because the treatment regimen did not meet the requirements of the study. Finally, of the 65 included patients, 31 patients were included in the AT group combined with first-line anti-angiogenesis therapy, and 34 patients were included in the CT group with first-line chemotherapy. 
a

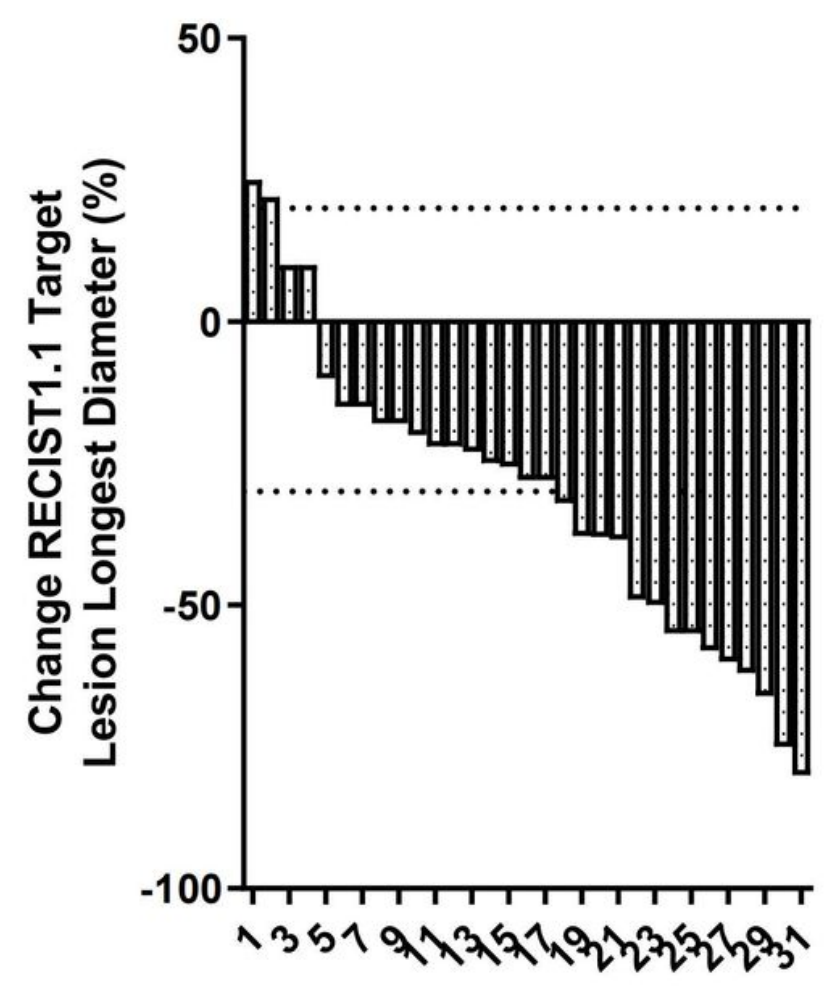

No. of Patients b

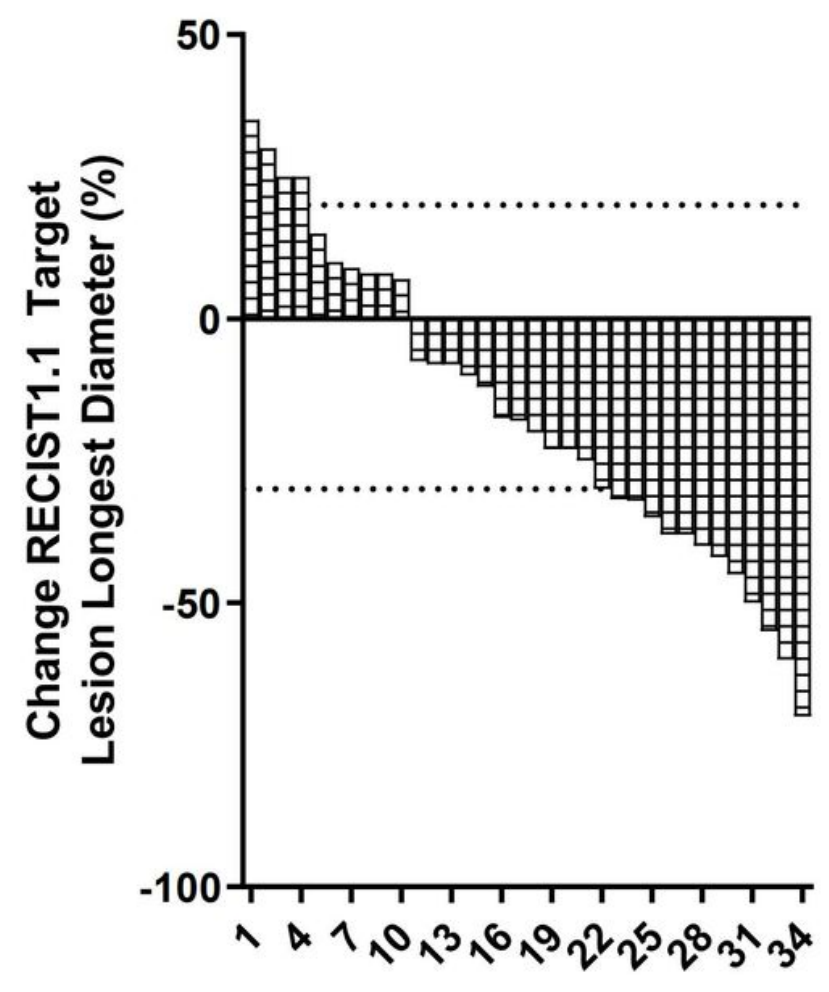

No. of Patients

Figure 2

Waterfall diagram of optimal efficacy evaluation for patients in the AT group and CT group. A) In the AT group, 15 patients achieved SD status, 14 patients achieved PR status, and 2 patients did not respond to treatment. B) In the CT group, 17 patients achieved SD status, 13 patients achieved PR status, and 4 patients did not respond to treatment. 


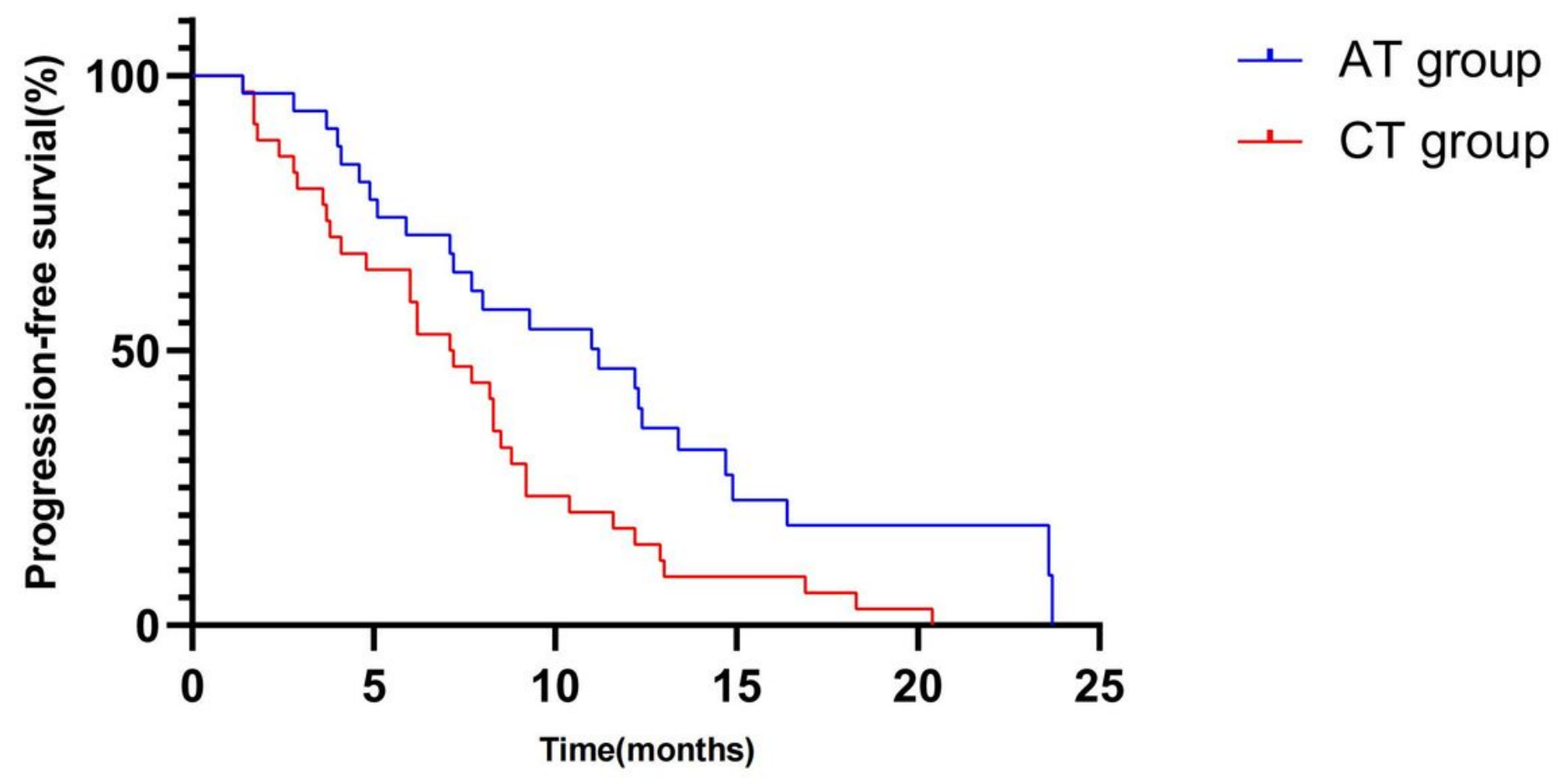

Figure 3

PFS in AT group and CT group. Median progression-free survival was 11.2 months in the AT group [95\% $\mathrm{Cl}, 5.9-16.5]$ and 7.0 months in the CT group [95\% Cl,5.1-8.9][HR, 0.49; 95\% Cl, 0.29-0.83; $\mathrm{P}=0.008]$. 

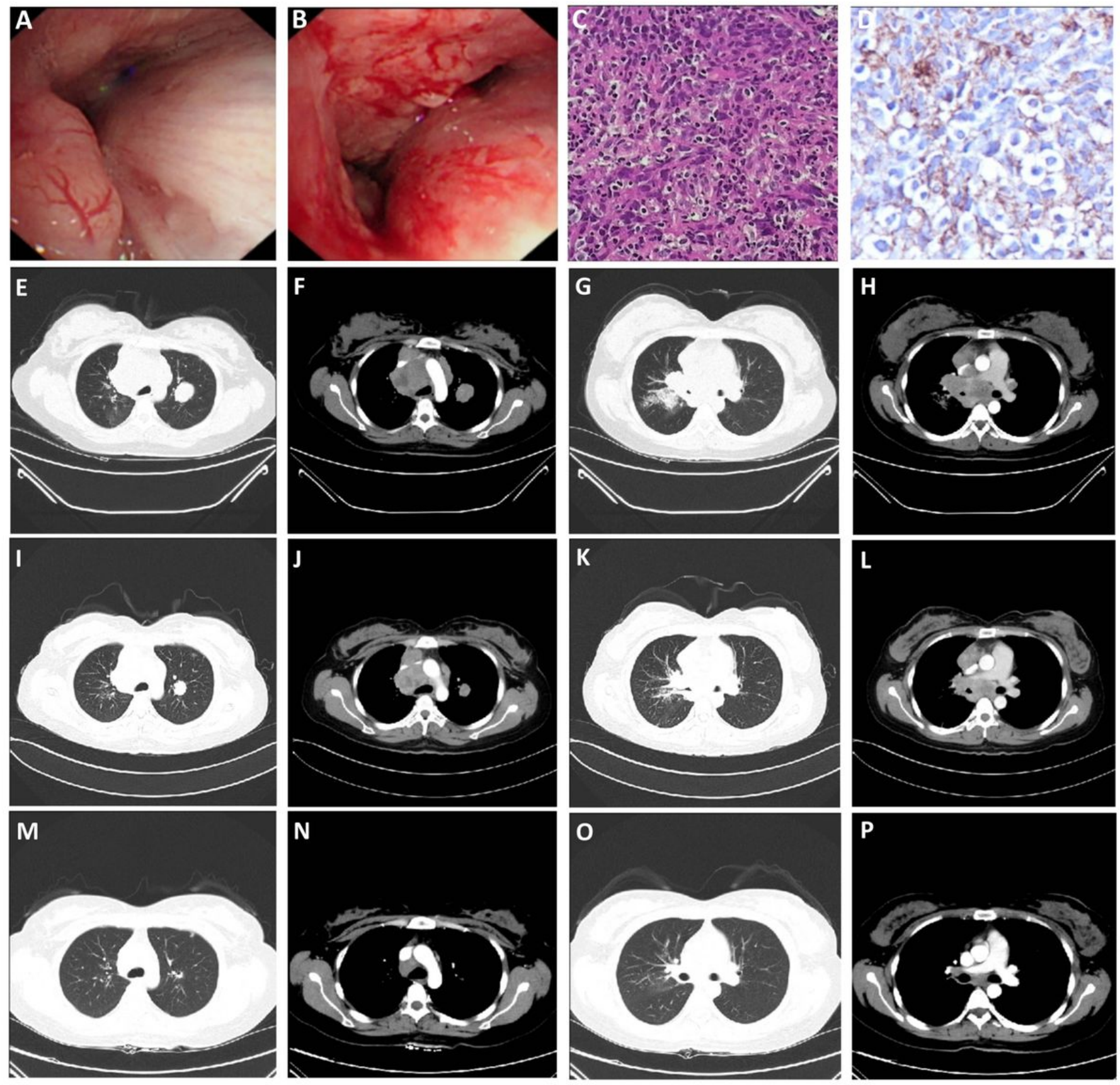

Figure 4

Radiographic images of the patient with FGFR3-TACC3 (F17:T10) fusion. A-B) Fiberbronchoscopy results in October 2018 indicated the growth of new organisms in the right main bronchus; C) HEx400 times, the results showed that some tumor cells were arranged in nests, with abundant cytoplasm, red staining, obvious nuclear atypia, interstitial fibrous hyperplasia accompanied by a large number of lymphocytes. D) Immunohistochemical ×400 times: PD-L1(22C3) CPS=80; E-H) Enhanced chest CT images in October 2018, mass soft tissue density shadow near hilum in the upper lobe of right lung, with obstructive pneumonia, multiple lymph node metastases in mediastinum and bilateral hilum, and double lung metastases ( $E$ and $\mathrm{G}$ pulmonary Windows, F and $\mathrm{H}$ mediastinal windows); I-L) Enhanced chest CT images in February 2019 showed that the mass soft tissue density shadow near hilum in the upper lobe of right lung was smaller than before, obstructive pneumonia was better than before, multiple lymph node metastases in mediastinum and bilateral hilum were smaller than before, and double lung metastases were smaller than before ( $\mathrm{I}$ and $\mathrm{K}$ lung Windows, $\mathrm{J}$ and L mediastinal Windows); M-P) Enhanced chest CT images in June 2019 showed that the mass soft tissue density 
shadow near hilum in the upper lobe of the right lung was smaller than before, obstructive pneumonia was better than before, multiple lymph node metastases in mediastinum and bilateral hilum were smaller than before, and double lung metastases were smaller and less than before ( $\mathrm{M}$ and $\mathrm{O}$ lung Windows, $\mathrm{N}$ and $\mathrm{P}$ mediastinal Windows). 